\title{
Facility for Heavy Ion Collision Experiment at RAON
}

\author{
Young Jin Kim¹,a, Do Gyun Kim¹, Gi Dong Kim¹, Yong Hak Kim¹, Young-Jin Kim¹, Yong Kyun \\ $\mathrm{Kim}^{1}$, Young Kwan Kwon ${ }^{1}$, Chong Cheol Yun ${ }^{1}$, Byungsik Hong ${ }^{2}$, Kyung Sei Lee ${ }^{2}$, Eun Joo Kim ${ }^{3}$, \\ Jung Keun $\mathrm{Ahn}^{4}$, and Hyo Sang Lee ${ }^{4}$ \\ ${ }^{1}$ Institute for Basic Science, Daejeon, Republic of Korea \\ ${ }^{2}$ Korea University, Seoul, Republic of Korea \\ ${ }^{3}$ Chonbuk National University, Jeonju, Republic of Korea \\ ${ }^{4}$ Pusan National University, Busan, Republic of Korea
}

\begin{abstract}
The Rare Isotope Science Project (RISP) was established in December 2011 in order to carry out the technical design and the establishment of the accelerator complex (RAON) for the rare isotope science in Korea. The rare isotope accelerator at RAON will provide both stable and rare isotope heavy-ion beams the energy range from a few $\mathrm{MeV} /$ nucleon to a few hundreds of $\mathrm{MeV} /$ nucleon for researches in fields of basic and applied science.

Large Acceptance Multipurpose Spectrometer (LAMPS) at RAON is a heavy-ion collision experimental facility for studying nuclear symmetry energy by using rare isotope beams. Two different experimental setups of LAMPS are designed for covering entire energy range at RAON.

One is for low energy ( $<18.5 \mathrm{MeV} /$ nucleon) heavy-ion collision experiment for day-1 experiments. This experimental setup consists of an array of $\Delta \mathrm{E}-\mathrm{E}$ Si-CsI detectors, a gamma array to cover backward polar angle, and a forward neutron wall.

The other is for completing an event reconstruction by detecting all the particles produced in high energy heavy-ion collisions within a large acceptance angle to measure particle spectrum, yield, ratio and collective flow of pions, protons, neutrons, and intermediate fragments at the same time. The experimental setup consists of a superconducting spectrometer, a dipole spectrometer, and a forward neutron wall. A Time Projection Chamber (TPC) will be placed inside of superconducting solenoid magnet of $0.6 \mathrm{~T}$ for charged particle tracking. The dipole spectrometer will be located forward of the superconducting spectrometer and it will be composed of a combination of quadrupole, dipole magnets, focal plane detector, tracking stations, and Time-of-Flight (ToF) detector at the end. The neutron wall will be made of 10 layers of plastic scintillators for neutron tracking.

In this presentation, the detail physics and design of LAMPS at RAON will be discussed.
\end{abstract}

\section{Introduction}

Rare Isotope beams (RIBs) are essential to understand not only the structure of exotic nuclei and the dynamics of heavy-ion collisions but also various nuclear astrophysics questions. In addition to this,

\footnotetext{
a e-mail: yjkim@ibs.re.kr
} 
RIBs can be used in several fields of applied science as well. The RIB accelerator and experimental facility in Korea, called RAON, will use both isotope separation on-line (ISOL) and in-flight fragmentation (IF) methods for RI production. For experiments using RIBs at RAON, there will be nuclear physics facilities for low and intermediate energies and applied facilities for mass measurement, laser spectroscopy, material science, neutron science, and bio-medical science. Details of RAON accelerator and experiments can be found in $[1,2]$.

There are more neutrons than protons in heavy stable nuclei. The semi-empirical mass formula successfully explains the masses of stable nuclei from light ones to heavy ones, but the structure of neutron-rich nuclei far from the limit of stability is not yet fully understood. Symmetry energy is the difference in energy per nucleon between pure neutron matter and symmetric nuclear matter $(N=Z)$. Therefore, understanding the nature of symmetry energy will offer clues to fundamental questions about the asymmetric constitution of nucleons in heavy nuclei, isospin asymmetry in nucleon-nucleon interactions, their modifications in a nuclear medium, etc.

Isospin-dependent nuclear reactions and the Equation of State (EoS) of isospin asymmetric nuclear matter are the contemporary research topics in nuclear physics that receive the most attention [3-5]. It is well known that the energy per nucleon of nuclear matter can be approximated by

$$
E(\rho, \delta)=E(\rho, \delta=0)+E_{\text {sym }}(\rho) \delta^{2}+O\left(\delta^{4}\right)
$$

where $\rho=\rho_{n}+\rho_{p}$ and $\delta=\left(\rho_{n}-\rho_{p}\right) / \rho$ are the total baryon density and the isospin asymmetry parameter, respectively, with $\rho_{n}\left(\rho_{p}\right)$ as the neutron (proton) density, $E(\rho, \delta=0)$ as the energy per nucleon of isospin symmetric nuclear matter, and $E_{\text {sym }}(\rho)$ as the bulk nuclear symmetry energy at the density $\rho$. In Equation (1) all odd-order $\delta$ terms are absent due to the charge symmetry of nuclear forces, and the quadratic or higher order terms in $\delta$ are negligible in most of cases. In particular, the determination of $E_{\text {sym }}$, which is coefficient of the isospin-dependent term of EoS or the density dependence of the nuclear symmetry energy, is the longstanding problem in nuclear physics and nuclear astrophysics. The Large Acceptance Multipurpose Spectrometer (LAMPS) at RAON is for studying nuclear symmetry energy of nuclear matter via heavy-ion collisions by using rare isotope beams. Details of physics and experimental setup will be discussed at following sections.

\section{Physics at LAMPS}

The physics goal at LAMPS is to understand the nature of symmetry energy in nuclei and in nuclear matter with nuclei from stable ones to those far from the stability valley, and to apply our knowledge to related phenomena from a microscopic scale to stellar objects. The RAON facility will provide high-intensity RIBs with unprecedented neutron-to-proton ratios. RAON will enable us to describe the quantitative distinction of various sources for the modification of the isospin-dependent EoS by measuring the energy (or density) dependence of several observables.

\subsection{Observables}

Physics observables at LAMPS experimental facility using both stable and RI beams at RAON are the following:

- Energy spectra of gammas from Pygmy/Giant dipole resonances

- Particle spectrum, yield, and ratio for isospin mirror particles (e.g. $\left.n / p,{ }^{3} \mathrm{H} /{ }^{3} \mathrm{He},{ }^{7} \mathrm{Li} /{ }^{7} \mathrm{Be}, \pi^{-} / \pi^{+}\right)$

- Collective flows (e.g. directed flow $\left(v_{1}\right)$, elliptic flow $\left(v_{2}\right)$ of $n, p$ and heavier clusters and azimuthal angle dependence of $n / p$ ratio with respect to reaction plane) 
- Isospin fractionation and isoscaling in nuclear multifragmentation

- Isospin diffusion.

It is important to measure the system size (different neutron-to-proton ratios), energy (available energies from lowest to maximum at RAON), centrality, rapidity and transverse momentum dependence of those observables to complete a systematic study of the symmetry energy in nuclear matter by bombarding RIBs on fixed targets.

\subsection{Experimental requirements}

In order to achieve its physics goal, the LAMPS experimental setup needs to measure various particles like $\pi^{ \pm}$, proton, neutron, heavy fragments from heavy-ion collisions at the same time for studying nuclear symmetry energy. Detector system requires to cover a large acceptance and to have excellent momentum/energy resolution for a variety of particle species with high efficiency. A gamma detector is also necessary for measuring Pygmy/Giant dipole resonances. In addition to the above requirement, flexibility of overall detector system is considered for other physics topics. Thus, two experimental setups are designed: One is at beam energy below $18.5 \mathrm{MeV} /$ nucleon for day-1 experiment and the other is at beam energy above 18.5 and up to $250 \mathrm{MeV} /$ nucleon.

\section{LAMPS experimental setup}

\subsection{Low-energy setup}

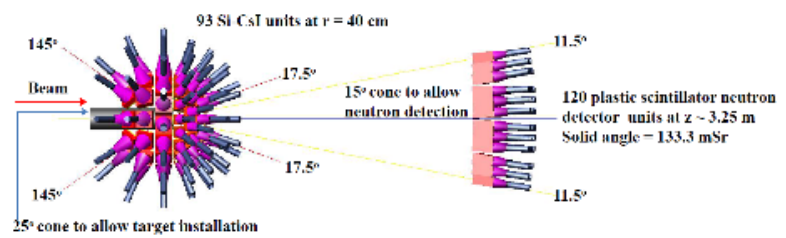

Figure 1. LAMPS low-energy experimental setup.

Figure 1 shows LAMPS low-energy experimental setup. All detector designs are based on GEANT-4 simulation [6] with simulated ${ }^{132} S n+{ }^{124} S n$ at $18.5 \mathrm{MeV} /$ nucleon collision events from Particle and $\underline{H}$ eavy Ion Transport code $\underline{S} y s t e m$ (PHITS) [7].

$93 \mathrm{Si}-\mathrm{CsI}(\mathrm{Tl})$ detectors are arranged in a 7 ring structure by estimation a particle hit probability below 0.1 within certain polar angle ranges. $9 \times 9 \mathrm{~cm}^{2}\left(15 \times 15 \mathrm{~cm}^{2}\right)$ of Si-CsI(Tl) detectors are used for polar angle range between $17.5^{\circ} \sim 77.5^{\circ}\left(78^{\circ} \sim 150^{\circ}\right)$ with $15^{\circ}\left(24^{\circ}\right)$ interval. A thickness of 100 $\mu \mathrm{m}$ for the Si detector was chosen for obtaining energy resolution of 0.5\% FWHM. For CsI(Tl) detector, $5 \mathrm{~cm}$ of thickness is especially selected not only for resolving charged particles (2\% of FWHM) but also measuring $30 \mathrm{MeV} \gamma$-ray from Pygmy and Giant dipole resonances. Charged particles from heavy-ion collisions can be measured and identified by the correlation between energy loss $(\Delta E)$ in $\mathrm{Si}$ detector and deposited energy $(E)$ in $\mathrm{CsI}(\mathrm{Tl})$ detector. In addition to this, the energy of gamma from Pygmy/Giant dipole resonances can be measured by $\mathrm{CsI}(\mathrm{Tl})$ detector.

Neutron detectors are designed in the same way as Si-CsI(Tl) detectors. Neutron detectors will be placed at a distance of $3.25 \mathrm{~m}$ from the target and the accuracy of measured neutron energy will be improved by measuring the time of flight information if neutron detector is longer than $20 \mathrm{~cm}$. 
GEANT-4 simulation shows that the detection efficiency of $60 \mathrm{MeV}$ neutrons is $80 \%$ for $20 \mathrm{~cm}$ long plastic scintillator. Particularly, the energy resolution is getting better to about $2.6 \%$ with time of flight information for the $20 \mathrm{~cm}$ long neutron detector case and energy resolution of $10 \mathrm{MeV}$ neutron is expected to be $\sim 3.3 \%$ with 500 ps time resolution and time of flight information.

\subsection{High-energy setup}

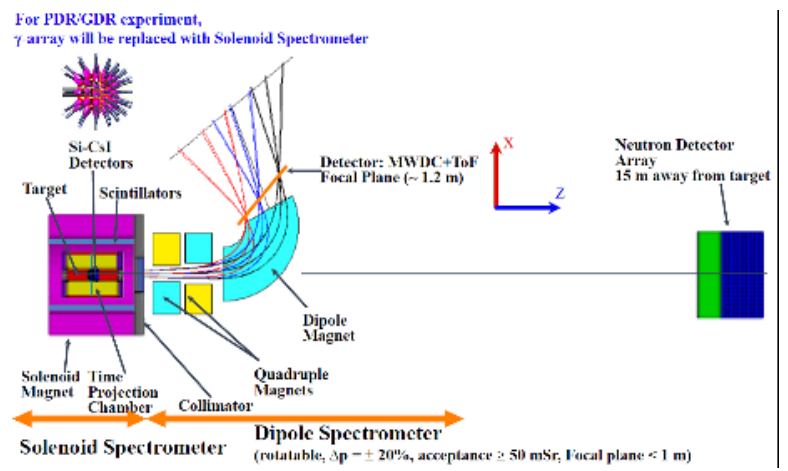

Figure 2. LAMPS high-energy experimental setup.

Figure 2 shows LAMPS high-energy experimental setup. It consists of three parts; a solenoid spectrometer, a dipole spectrometer, and a forward neutron array. A GEM based cylindrical Time projection chamber (TPC, $1 \mathrm{~m}$ diameter $\times 1.2 \mathrm{~m}$ length) is located inside a $0.6 \mathrm{~T}$ solenoid magnet as main charged particle tracking detector and it will give us complete information of particle momentum and identification from track reconstruction. Plastic scintillators will surround the TPC from outside and they will be used for trigger and time of flight information of charged particles which will enhance the capability of particle identification. Si-CsI will be placed inside the TPC cylinder $\left(14^{\circ}<\right.$ polar angle $<24^{\circ}$ ) and will measure low momentum charged particles.

The dipole spectrometer consists of two quadruple magnets, a dipole magnet, and a focal plane detector to measure intermediate fragments. For focal plane detector, combination of MWDC and time of flight detectors are under consideration.

The neutron detector array will be located very forward $15 \mathrm{~m}$ away from target position for neutron tracking. The neutron detector array consists of one layer of charged particle veto and 10 layers of neutron detectors.

\section{References}

[1] RISP, Few-Body Systems 54, 961 - 966 (2013)

[2] RISP, Nuclear Instruments and Methods in Physics Research B, to be published

[3] Bao-An Li, Lie-Wen Chen, and Che Ming Ko, Physics Reports 464, 113 - 281 (2008)

[4] P. Danielewics, R. Lacey, and W. G. Lynch, Science 298, 1592 - 1596 (2002)

[5] J. M. Lattimer and M. Prakash, Science 304, 536 - 542 (2004)

[6] S. Agostinelli et al, Nuclear Instruments and Methods in Physics Research A 506, 250 - 303 (2003)

[7] T. Sato et al, Journal of Nuclear Science and Technology 50, 913 - 923 (2013) 